\title{
Analysis of 4G mobile network coverage in UTeM technology campus
}

\author{
Zahariah Manap $^{1}$, Anis Suhaila Mohd Zain ${ }^{2}$, Rahaini Mohd Said ${ }^{3}$, Shawn Shivaneson Balakirisnan ${ }^{4}$ \\ ${ }_{1,3,4}$ Faculty of Electrical and Electronic Engineering Technology (FTKEE), \\ Universiti Teknikal Malaysia Melaka (UTeM), Malaysia \\ ${ }^{2}$ Faculty of Electronics and Computer Engineering (FKEKK), Universiti Teknikal Malaysia Melaka (UTeM), Malaysia
}

\begin{tabular}{l} 
Article Info \\
\hline Article history: \\
Received Aug 25, 2019 \\
Revised Nov 26, 2019 \\
Accepted Dec 10, 2019 \\
\hline
\end{tabular}

\section{Keywords:}

$4 \mathrm{G}$

Network coverage

RSSI

Signal propagation

\begin{abstract}
This paper proposes an analysis of the coverage performance of $4 \mathrm{G}$ cellular services in UTeM Technology Campus. The performance of the cellular services is presented as the network's coverage profile which is based on the received signal strength indicator (RSSI). The area under study is virtually divided into 64 grid points where the average RSSI measurements are captured by using an open source software namely G-Mon. The measured values are mapped into the network coverage profile which represents the signal reception quality at each of the grid points. A statistical analysis called Two-Way ANOVA is performed to investigate the correlation of the performance of $4 \mathrm{G}$ cellular services in UTeM Technology Campus with the mobile phone brands and service operators. Based on the analysis, it is found that the signal reception in outdoor areas are better than that of indoor areas. In addition, the analysis shows that the propagation loss and signal degradation are two factors that contribute to the $4 \mathrm{G}$ services' performance in UTeM Technology Campus.
\end{abstract}

Copyright $\odot 2020$ Institute of Advanced Engineering and Science. All rights reserved.

\section{Corresponding Author:}

Zahariah Manap,

Department of Electronics and Computer Engineering Technology,

FTKEE, UTeM, Hang Tuah Jaya, 76100 Durian Tunggal, Melaka, Malaysia.

Email: zahariah@utem.edu.my

\section{INTRODUCTION}

One of the key aims of modern telecommunication networks is to deploy high-capacity coverage over a wide area. Since it was introduced in 1970's [1], the mobile cellular communication technology has evolved dramatically. Starting with the First Generation (1G) in 1980's, with a very low data rate and small coverage, the systems have been improved rapidly until the current technology known as the Fourth Generation (4G) or Long Term Evolution (LTE) which provides much higher data rate and larger network coverage [2-5]. The specifications and performance requirements of LTE are standardized by the 3rd Generation Partnership Project (3GPP). The standard was developed based on the two preceding technologies which are Global System for Mobile (GSM) and Universal Mobile Telecommunication Systems (UMTS).

The main feature of LTE is to provide cheaper spectrum efficiency for the operators, as well as mobile broadband services with high quality to users [6-7]. It is aimed to provide global mobile access and good fidelity of data, voice, audio, and video transmissions. The users must be reachable wherever they are and at the same time while they move, the communications must not be interrupted [8]. One of the challenges in offering the wide coverage is the vulnerability of the transmission medium due to signal attenuation, blocking, scattering and reflection. The wireless channels are prone to noise and error due to the signal propagation mechanisms which limit the network coverage especially in indoor environments and highdensity areas. 
Several works have been performed to analyse the performance of cellular network coverage in outdoor [4-5], indoor [9-12] and outdoor-indoor environments [13-18]. The analysis from previous works shows that the network coverage performance in indoor environments differs from that of outdoor environments. While the outdoor signal propagations are easier to be estimated, indoor coverage estimation needs an accurate physical information of the site such as the construction materials and furniture arrangement. Therefore, predicting the received signal strength (RSS) for a mobile station (MS) located inside a building based on an outdoor propagation model is impractical. In that respect, a better network planning for a specific area especially in indoor environments can be achieved by capturing and analysing the real RSS measurements in that particular area.

The main objective of this paper is to investigate the 4G cellular networks' signal quality in UTeM Technology Campus. The signal quality is presented as the network's coverage profile which was generated based on the received signal strength indicator (RSSI). The RSSI measurements were obtained through an open source software namely G-Mon which was installed on two different mobile phone's brands. The area under study is virtually divided into 64 grid points where the average readings were captured at each of the points and mapped into the network coverage profile. Also, a statistical analysis was carried out to investigate the correlation of the quality of signal reception between mobile phone brands and service operators.

The rest of this paper is organized as follows. Section II reviews the mobile radio wave propagation and Section III describes the methodology that has been carried out in this work. The results and discussion are presented in Section IV. Finally, Section V draws the conclusion of the work.

\section{MOBILE RADIO WAVE PROPAGATION}

Generally, the celllular radio propagation models can be categorized into two main classes which are outdoor and indoor propagation models. The performance of $4 \mathrm{G}$ cellular networks at any distance from the BS relies on the quality of the signal reception. Good signal reception guarantees higher throughput and continuous connectivity. The most basic cellular radio propagation model is the free space propagation model that describes the relationship between the RSS and transmitter-receiver (T-R) separation. The RSS at a specific distance, $\mathrm{d}$ is given by (1).

$$
P_{r}(\mathrm{~d})=\frac{P_{t} G_{t} G_{r} \lambda^{2}}{(4 \pi)^{2} d^{2}}
$$

where $P_{t}$ is the transmitted power, $G_{t}$ is the transmitter antenna gain, $G_{r}$ is the receiver antenna gain, $\lambda$ is the wavelength in meters and $d$ is the T-R separation in meters. While (1) gives a rough signal reception prediction, real measurement should also consider many other factors such as the terrain and landscape characteristics in which the MS and base station (BS) are located. The free space propagation model considers only free space condition where there are no obstacles between the BS and MS. A better and accurate propagation models are needed to simulate the effects of the surrounding obstacles. Moreover, rapid demand for cellular radio communications has forced the continuous enhancement study on the radio propagation in cellular networks to verify the wireless channel performance according to technology requirements.

In the environments that are rich with obstacles and scatterers, the transmitted signals will experience reflection, diffraction and scattering which affect the RSS [19]. The Okumura-Hata and COST231 are two empirical models that describe the signal propagation in different clutter type environments more accurately. The Okumura-Hata model gives the estimation of mobile signal's path loss in high-density areas for the carrier frequency of $700 \mathrm{MHz}$ to $1500 \mathrm{MHz}$. The median path loss calculation based on Okumura-Hata Model is given by (2):

$$
L_{50}(d B)=69.55+26.6 \log _{10} f_{c}-13.82 \log _{10} h_{t e}-\alpha\left(h_{r e}\right)+\left(44.9-6.55 \log _{10} h_{t e}\right) \log _{10} d
$$

where the terms $f_{c}, h_{t e}$ and $h_{r e}$ represent the carrier frequency, transmitter antenna height and receiver antenna height respectively. The term $\alpha\left(h_{r e}\right)$ is the correction factor for MS antenna which is calculated according to the clutter type such as urban, sub-urban and countryside.

The COST-231 model is an extension of the Okumura-Hata model which covers the carrier frequencies that range from $1500 \mathrm{MHz}$ to $3500 \mathrm{MHz}$. This model is more accurate as it consists of a correction constant for urban, suburban and rural environments. The median path loss calculation based on the COST231 model is given by (3):

$$
L_{50}(d B)=46.3+33.9 \log _{10} f_{c}-13.82 \log _{10} h_{t e}+c_{m}-\alpha\left(h_{r e}\right)+\left(44.9-6.55 \log _{10} h_{t e}\right) \log _{10} d
$$


The notations used in (3) are similar to that of (2), while the correction constant, $\mathrm{cm}$ is defined as 0 $\mathrm{dB}$ for the case of suburban or open environments and $3 \mathrm{~dB}$ for the case urban environments.

The original Okumura-Hata model was developed based on the measurement campaign carried out in the city of Tokyo, Japan. Therefore, the model specifically represents the radio signal profile in the city. Despite this model can be used for radio signal prediction in other cities, it requires a correction to suit the environmental characteristics of that particular city. Numerous works have used Okumura-Hata model as the basis to predict the radio signal strength in different areas and frequency bands. The authors in [20] proposed a site-specific propagation model based on Okumura-Hata model to predict $900 \mathrm{MHz}$ radio signals in Egypt. The proposed model shows an improvement in the signal strength prediction when compared to the original model. Similar works are also found in [21-22] which consider an area in Nigeria and Cameroon respectively. Apart from those works, a meticulous study was carried out to investigate the effect of spatial and time changes ratios in the measurement of wave propagations in indoor and outdoor environments [23]. Based on the measurement results, two propagation models were proposed as the enhancement of the free space and COST-231 model.

In the case where the BS is located outside the buildings, the propagation of the wireless signal from outdoor to the indoor is influenced by the building's wall structure and indoor furniture (scatterers) arrangement $[16,24]$. The propagation model of the signal transmitted from an outdoor BS to an MS located in a building is described in Figure 1. The outdoor-to-indoor propagation loss is calculated by using the COST-231 Building Penetration LOS model [17, 25] as in (4):

$$
L_{50}(d B)=L_{f}(S+d)+W_{e}+W G_{e}(1-\cos \theta)^{2}+\alpha(d-2)(1-\cos \theta)^{2}
$$

where $L_{f}(\cdot)$ is the free space propagation loss between the BS and MS, and $\alpha$ is the indoor attenuation constant. The term $\mathrm{S}$ and d denotes the distance between the BS and the wall penetration point and the distance between the wall and the MS respectively. The symbol $\theta$ represents the angle between the direction of signal's arrival and the normal line of the wall at the point of penetration. The term We and WGe represents the loss of the external wall when $\theta$ is 0 o and when $\theta$ is 90 o respectively.

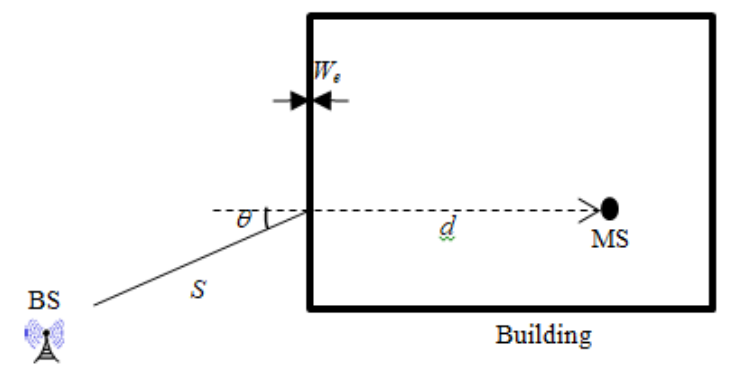

Figure 1. The COST-231 Building Penetration LOS model

\section{METHODOLOGY}

The flowchart in Figure 2 describes the overall process of capturing and analysing the performance of $4 \mathrm{G}$ cellular signals in UTeM Technology Campus. The analysis is started by selecting the area under study. As shown in Figure 3, the area of study encloses 6 buildings which are Fakulti Teknologi Kejuruteraan (FTK) building, Fakulti Kejuruteraan Mekanikal (FKM) building, cafeteria, Factory 1, Factory 2 and Factory 3 , and two car park areas. With the aid of the map plan obtained from Google Earth, the area under study is sectorized into 64 grid points as depicted in Figure 3.

The RSSI of $4 \mathrm{G}$ cellular signal at each of the grid points were measured by using an open software named G-Mon which was run on two different brands of mobile phone. By considering the available resources, a Xiaomi Redmi 3S and Asus Zenfone 4 are used in this work. Also, only two service providers are considered in this work which are Digi and U-Mobile. Four datasets are recorded by using four mobile phone brand-service provider combinations; Xiaomi Redmi 3S-Digi, Xiaomi Redmi 3S-U-Mobile, Asus Zenfone 4-Digi and Asus Zenfone 4-U-Mobile. 


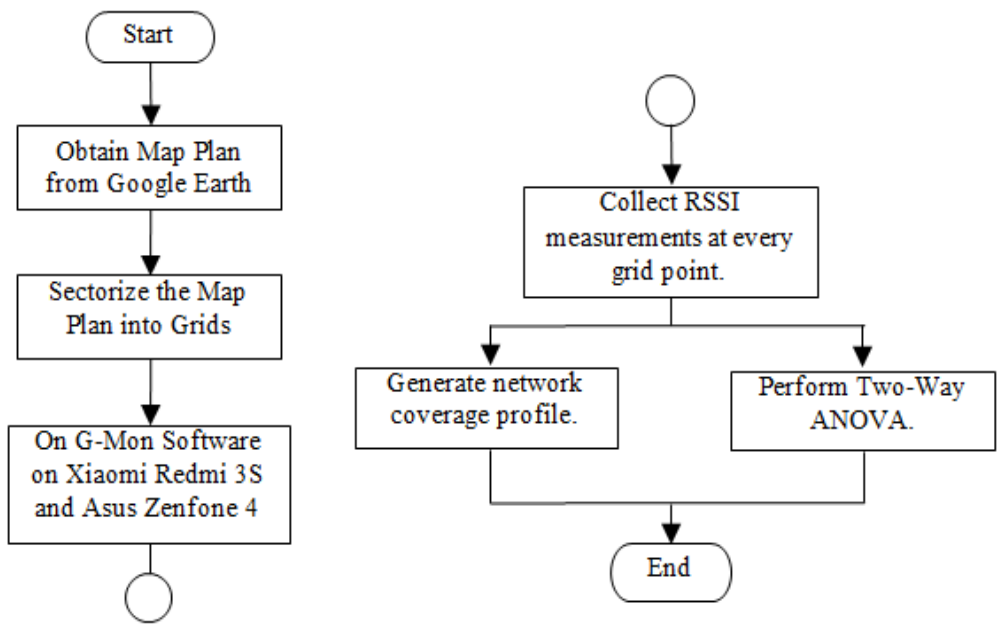

Figure 2. The flowchart of the methodology

Based on the measured data, the $4 \mathrm{G}$ network coverage is generated by mapping the RSSI values by referring to the standard RSSI level index in Table 1. Finally, a two-way ANOVA is used to investigate the correlation of the signal reception between mobile phone brands and service providers.

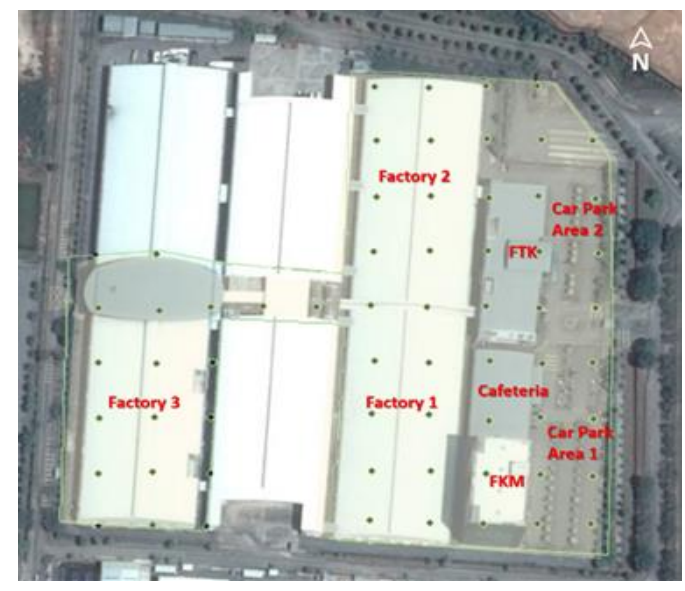

Figure 3. The map plan of the area under study

Table 1. The RSSI Level Index

\begin{tabular}{cc}
\hline Signal Strength Description & RSSI $(\mathrm{dBm})$ \\
\hline Excellent & $>-65$ \\
Good & -65 to -75 \\
Fair & -75 to -85 \\
Poor & $<-85$ \\
\hline
\end{tabular}

\section{RESULT AND DISCUSSION}

The results obtained from the measurement series are analyzed by using two methods. The first analysis shows the network performance in visual representation based on the RSSI level index. The second analysis was carried out by a statistical mean called Two-Way ANOVA.

\subsection{Network Coverage Profile}

The measured RSSI for the four datasets are presented in terms of network coverage profile as depicted in Figure 4(a) through Figure 4(d). From the figures, it can be grossly seen that all datasets strongly agree to each other by producing an almost similar profile pattern. The profile patterns show that the 
performance of $4 \mathrm{G}$ cellular signal in the outdoor area of the two car parks ranges from excellent to good RSSI, while FKM building, cafeteria and FTK building have good RSSI. For the indoor area of Factory 3, it can be said that the overall $4 \mathrm{G}$ cellular signal's performance is fair, with some parts of the area have poor signal reception. On the contrary, the indoor areas of Factory 1 and Factory 2 show very poor performance where the RSSI at almost of the grid points in these two buildings is recorded less than $-85 \mathrm{dBm}$. Also, the same pattern is observed along the walkway heading Factory 3.

It is obvious to see from the network coverage profile that the $4 \mathrm{G}$ cellular services are very excellent in outdoor environments as the probability of shadowing effect is low. While in indoor environments, the performance varies depending to the building's location and structure.

\subsection{Statistical Analysis}

The statistical mean analysis is carried out to further investigate the factors that lead to the resulted network coverage profile. For that purpose, a Two-Way ANOVA was performed to examine the correlation between all four datasets. The intention of the analysis is to test whether service providers significantly affect the signal reception quality in UTeM Technology Campus. Furthermore, the analysis also investigates the effect of using different brand of mobile phones. The null hypothesis of the test says that the mean of the two data groups is similar.

Table 2 summarizes the ANOVA table of the test. The result in the first row of the table shows the correlation between two different groups of samples which represents two different brands of mobile phone. The $\mathrm{p}$-value of 0.868878 is greater than the critical value $(0.05)$. This suggests that the null hypothesis must be accepted. In other words, it is said that there is no significant effect of using different brands of mobile phone on the signal reception quality. Similarly, the p-value yielded when two different service providers are compared is 0.464817 which is greater that the predetermined critical value. Thus, it is said that the mean of the data of the both groups has a small difference, leading to the conclusion that using different service providers merely affect network coverage.
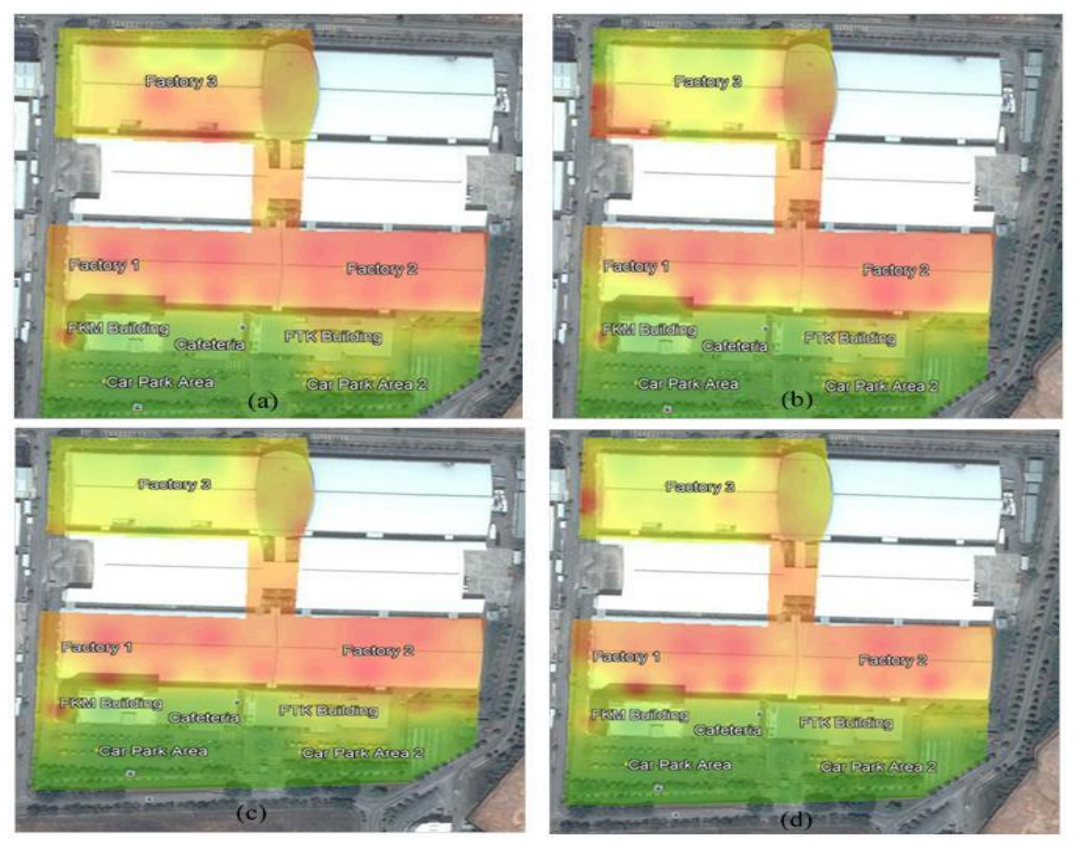

Figure 4. Mobile Network coverage profile (a) U Mobile (Xiaomi Redmi 3s), (b) U Mobile (Asus Zenfone 4), (c) Digi (Xiaomi Redmi 3s), (d) Digi (Asus Zenfone 4)

The fourth row of the table describes the interaction of the data measured by using two different brands of mobile phone and the service providers. Once again, the test confirms that there is no significant difference of using different brand of mobile phone while subscribing to any of the two services. From the network coverage profile in Figure 4(a) through Figure 4(d), it can be said that the mobile signal quality in UTeM Technology Campus is divided into four zones involving outdoor and indoor environments as summarized in Table 3. 
The observation is validated by the statistical analysis of the data which conclude that the zoning of signal reception is independent of either service providers or brand of the mobile phones. Zone 1 covers the two car park areas which are outdoor environments. The excellent signal reception in Zone 1 portrays the line of sight (LOS) condition where the mobile signals transmitted by the nearby BSs can easily reach the open areas where no major obstacles present. Zone 2 which has good signal reception covers FTK building, FKM building and Cafeteria. Since Zone 2 is an indoor environment, the reception in this zone is worse than that of Zone 1 due to the non-line of sight (NLOS) signal propagation. Conversely, Zone 3 and Zone 4 are also affected by the NLOS signal propagation. However, the reception in these zones is worse when compared to that of Zone 2. This suggests that apart from NLOS signal propagation, the design structure of the building is another factor that affect the network coverage quality.

Table 2. Comparison between Two Different Smartphones under U Mobile Network using ANOVA

\begin{tabular}{ccccccc}
\hline Source of Variation & SS & df & MS & F & P-value & F crit \\
\hline Sample (Handphone brands) & 2.977781641 & 1 & 2.977781641 & 0.027308 & 0.868878 & 3.878624 \\
Columns (Service providers) & 58.43646914 & 1 & 58.43646914 & 0.535901 & 0.464817 & 3.878624 \\
Interaction & 86.08360352 & 1 & 86.08360352 & 0.789443 & 0.375116 & 3.878624 \\
Within & 27478.95691 & 252 & 109.0434798 & & & \\
Total & 27626.45477 & 255 & & & & \\
\hline
\end{tabular}

Table 3. The Zones of 4G Signal Reception Quality

\begin{tabular}{cc}
\hline Zone & Area \\
\hline Zone 1 (Excellent) & Car park areas (outdoor) \\
Zone 2 (Good) & FTK, FKM, Cafe (indoor) \\
Zone 3 (Fair) & Factory 3 (indoor) \\
Zone 4 (Poor) & Factory 1, Factory 2 (indoor) \\
\hline
\end{tabular}

Based on the observation, it can be said that the poor 4G signal reception in the factory buildings is due to the location and the buildings' design structure. The design structure of the factory buildings differs from the FTK and FKM buildings in such a way that the two buildings are tall and surrounded with glass walls whilst the factories are the lowest yet widest buildings in the area. This factor prohibits the penetration of direct signals into the buildings. In addition, the buildings' interiors are rich with wall structures that divide the space into partitions and furniture that act as scatterers. The walls and scatterers are the agents that promotes the occurrence of multipath or fast fading due to destructive interference of multiple reflected signals received by the mobile phones.

\section{CONCLUSION}

This paper measure and analyze the RSSI of $4 \mathrm{G}$ signals provided by two service operators in UTeM's Technology. The objective of the paper is to show the network coverage profile of the two operators and carry out initial investigation of the factors that contribute to the signal reception quality in the campus. The network coverage profiles produced from four datasets show that the area is divided into four zones based on the signal reception quality which are excellent, good, fair and poor areas. In addition, it is prominent to see that the signal reception quality in outdoor area is much better than that of indoor area. Based on the analysis, we suggest that propagation loss and signal degradation due to the present of obstacles contribute to the 4G services' performance. The findings in this paper will be the basis of our future works which will be focusing on analyzing the impacts of penetration loss on the RSS in indoor environments.

\section{ACKNOWLEDGEMENTS}

The authors wish to acknowledge Universiti Teknikal Malaysia Melaka (UTeM) and the Malaysia Ministry of Higher Education for the financial funding under Grant No. RACE/F3/TK3/FKEKK/F00299 and providing instrumentation devices support for this project.

\section{REFERENCES}

[1] L. Song and J. Shen, "Evolved cellular network planning and optimization for UMTS and LTE". 2010.

[2] E. Dahlman, J. Skold, and S. Parkvall, "4G, LTE-advanced and the Road to 5G", Third Edit. Academic Press, 2016.

[3] L. Zhang et al., "Propagation modeling for outdoor-to-indoor and indoor-to-indoor wireless links in high-speed train," Meas. J. Int. Meas. Confed., vol. 110, pp. 43-52, 2017. 
[4] H. Xu, C. Shi, W. Zhang, and Y. Yang, "Field testing, modeling and comparison of multi frequency band propagation characteristics for cellular networks," in IEEE International Conference on Communications, ICC 2016, 2016, pp. 1-5.

[5] S. K. Jha, R. Rokaya, A. Bhagat, A. R. Khan, and L. Aryal, "LTE Network: Coverage and Capacity Planning - 4G Cellular Network Planning around Banepa," in International Conference on Networking and Network Applications (NaNA), 2017, pp. 180-185.

[6] E. T. Tchao, J. D. Gadze, and J. O. Agyapong, "Performance evaluation of a deployed 4G LTE network," Int. J. Adv. Comput. Sci. Appl., vol. 9, no. 3, pp. 165-178, 2018.

[7] Z. Bo, D. Yuanfeng, and Y. Dongkai, "The Impact of New Features on Positioning Technology in LTE-A System," Mob. Inf. Syst., vol. 2015, pp. 1-10, 2015.

[8] J. Walrand and S. Parekh, "Communication Networks: A Concise Introduction", in Synthesis Lectures on Communication Networks, vol. 3, no. 1, 2010, pp. 1-192.

[9] L. Zhang, X. Gu, Z. Liu, L. Zhang, and H. Moon, "Modeling and Analysis of Indoor Coverage Probability for Future 3D Dense Mobile Networks," in International Symposium on Wireless Personal Multimedia Communications, 2017, pp. 246-251.

[10] A. A., A. Y. K., U. N.M, and A. E.E, "Radio Wave Detection Using Cost 231-Hata Model for Wireless Network Planning; A Case Study of Senate Building Environs of Unilag, Nigeria," Int. J. Adv. Sci. Res. Eng., vol. 4, no. 12, pp. 107-113, 2018.

[11] E. Vinogradov, A. Bamba, W. Joseph, and C. Oestges, "Physical-Statistical Modeling of Dynamic Indoor Power Delay Profiles," IEEE Trans. Wirel. Commun., vol. 16, no. 10, pp. 6493-6502, 2017.

[12] J. Senic, C. Gentile, P. B. Papazian, K. A. Remley, and J. K. Choi, “Analysis of E-Band Path Loss and Propagation Mechanisms in the Indoor Environment," IEEE Trans. Antennas Propag., vol. 65, no. 12, pp. 6562-6573, 2017.

[13] M. K. Marina, V. Radu, and K. Balampekos, "Impact of Indoor-Outdoor Context on Crowdsourcing based Mobile Coverage Analysis," in Workshop on All Things Cellular: Operations, Applications and Challenges, 2015, pp. 45-50.

[14] F. Afroz, R. Subramanian, R. Heidary, K. Sandrasegaran, and S. Ahmed, "SINR, RSRP, RSSI and RSRQ Measurements in Long Term Evolution Networks," Int. J. Wirel. Mob. Networks, vol. 7, no. 4, pp. 113-123, 2015.

[15] S. Ahmad, S. Musleh, and R. Nordin, "The Gap between Expectation \& Reality: Long Term Evolution (LTE) \&Third Generation (3G) Network Performance in Campus with Test Mobile System (TEMS)," in International Conference on Mathematical Modelling and Computer Simulation, 2016, pp. 164-168.

[16] V. Degli-Esposti, E. M. Vitucci, and R. Martin, "A Simple and Versatile Field Prediction Model for Indoor and Indoor-to-Outdoor Propagation,” IEEE Access, vol. 5, pp. 13270-13276, 2017.

[17] G. Castro, R. Feick, M. Rodríguez, R. Valenzuela, and D. Chizhik, "Outdoor-to-Indoor Empirical Path Loss Models: Analysis for Pico and Femto Cells in Street Canyons," IEEE Wirel. Commun. Lett., vol. 6, no. 4, pp. 542-545, 2017.

[18] V. Degli-Esposti et al., "A Semideterministic Model for Outdoor-to-Indoor Prediction in Urban Areas," IEEE Antennas Wirel. Propag. Lett., vol. 16, pp. 2412-2415, 2017.

[19] R. Nlend and E. Tonye, "Planning and simulation of LTE radio network: case of the city of Yaoundé," J. Electron. Commun. Eng., vol. 14, no. 2, pp. 19-29, 2019.

[20] M. Farhoud, A. El-Keyi, and A. Sultan, "Empirical correction of the Okumura-Hata model for the $900 \mathrm{MHz}$ band in Egypt," in International Conference on Communications and Information Technology, 2013, pp. 386-390.

[21] Z. K. Adeyemo, O. K. Ogunremi, and I. A. Ojedokun, "Optimization of okumura-hata model for long term evolution network deployment in lagos, nigeria," Int. J. Commun. Antenna Propag., vol. 6, no. 3, pp. 146-152, 2016.

[22] D. Djomadji Eric Michel and T. Emmanuel, "Optimization of Okumura Hata Model in 800MHz based on Newton Second Order algorithm. Case of Yaoundé, Cameroon,” J. Electr. Electron. Eng., vol. 10, no. 2, pp. 2278-1676, 2015.

[23] S. K. Noh and D. Y. Choi, "Propagation model in indoor and outdoor for the LTE communications," Int. J. Antennas Propag., vol. 2019, 2019.

[24] W. Xue, W. Qiu, X. Hua, and K. Yu, "Improved Wi-Fi RSSI Measurement for Indoor Localization," IEEE Sens. J., vol. 17, no. 7, pp. 2224-2230, 2017.

[25] N. S. Nkordeh, A. A. A. Atayero, F. E. Idachaba, and O. O. Oni, "LTE network planning using the Hata-Okumura and the COST-231 Hata pathloss models," Lect. Notes Eng. Comput. Sci., vol. 1, pp. 705-709, 2014.

\section{BIOGRAPHIES OF AUTHORS}

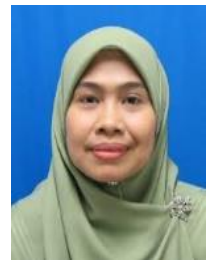

Zahariah binti Manap is currently working as a senior lecturer in Department of Electronics and Computer Engineering Technology, Faculty of Engineering Technology, Universiti Teknikal Malaysia Melaka. She received her B.E and M.E. in Communication and Computer Engineering from Universiti Kebangsaan Malaysia, Selangor, Malaysia in 2000 and 2003 respectively. Her research interests include Mobile \& Wireless Communications, Wireless Sensor Networks and Positioning \& Location Estimation. 


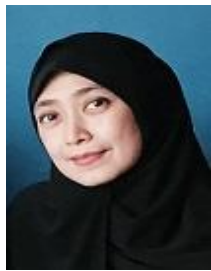

Anis Suhaila Binti Mohd Zain (CEng) received the Master degree in Microelectronics from National University of Malaysia in 2001, and the Ph.D. degree in Electronics and Electrical Engineering from the University of Glasgow, Glasgow, U.K., in 2013. She is currently senior lecturer in Faculty of Electronic and Computer Engineering (FKEKK), Universiti Teknikal Malaysia Melaka (UTeM). She has the research interest in nanoscale device design and simulation, statistical variability and reliability, and IC design for biomedical applications.

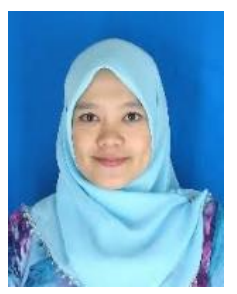

Rahaini Mohd Said was born in Johor, Malaysia. She is a Statistics and Mathematics lecturer of Faculty of Engingeering Technology, Universiti Teknikal Malaysia Melaka. Her first Degree was Mathematical Science majoring in Statistics from Universiti Teknologi Mara in 2009, then completed her MSc in Mathematical Science in 2011 from Universiti Teknologi Malaysia. She has published numerous academic papers in journal and conference related to applied statistics and other related areas. Her current main interests of research are Applied Statistics, Experimental Design and Data Science Specifically in Machine Learning.

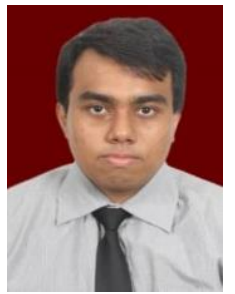

Shawn Shivaneson M Balakirisnan is currently a student at Faculty of Engineering Technology, Universiti Teknikal Malaysia Melaka. He is majoring in Telecommunication Engineering Technology. His research interest in Mobile \& Wireless Communications. 